\title{
Research Progress on the Relationship between Polymor- phism and SLE of Vitamin D Metabolic Pathway Related Gene
}

\author{
${\text { Saijia } \mathrm{Li}^{\mathrm{a}}}$ Zhilu Li $^{\mathrm{a}}$ Yiyu Cai ${ }^{\mathrm{b}}$ Guanlu $\mathrm{Li}^{\mathrm{c}}$ Xiaomin Zhuang ${ }^{\mathrm{d}}$ Tengxiao Chu ${ }^{\mathrm{a}}$ Daifeng Zhou ${ }^{\mathrm{e}^{*}}$ \\ Hainan Medical University a.The First Clinical College; b.College of Tropical Medicine and Laboratory Medicine; \\ c.College of Public Health; d.College of Management; e.College of Basic Medicine and Life Sciences, Haikou, Hainan, \\ 570100 , China
}

\begin{abstract}
Vitamin D is a class of hormones necessary to maintain normal physiological activities of the body. A large number of studies have shown that vitamin $\mathrm{D}$, as a fat soluble vitamin, is not only related to calcium and phosphorus metabolism, but also closely related to immune regulation, humoral regulation, cell cycle and so on.Systemic Lupus erythematosus (SLE) is a specific autoimmune diffuse connective tissue disease that causes tissue and organ damage under the joint action of multiple factors such as environment and heredity. Among many factors, the vitamin D metabolism pathway gene is particularly important for its influence. Several literatures have shown that the genetic polymorphism of vitamin D metabolic pathway genes is correlated with SLE.Therefore, by referring to relevant literature, this paper summarized the progress in the research on the mechanism of genetic polymorphism of vitamin metabolism pathway genes and the development of SLE.
\end{abstract}

\section{Keywords}

Vitamin D metabolic pathways; gene polymorphism; systemic lupus erythematosus

\section{维生素D 代谢通路单核苷酸多态性与系统性红斑狼疮的关联 性研究进展}

\section{李赛嘉 ${ }^{a}$ 李志路 ${ }^{a}$ 蔡奕渔 $^{b}$ 黎冠陆 $^{c}$ 庄小敏 ${ }^{d}$ 褚腾霄 ${ }^{a}$ 周代锋 $\mathrm{e}^{*}$}

海南医学院 a. 第一临床学院; b. 热带医学与检验医学院； c. 国际教育学院；d. 管理学院； e. 基础医学与生命科学学院，中国・海 南海口 570100

\section{摘 要}

维生素 D 是维持机体正常生理活动的必需维生素。大量研究表明, 维生素 D 作为脂溶性维生素, 除钻磷代谢经典效应 外, 在机体的免疫调节、抗炎症反应、细胞增殖等方面的作用日受到研究者的关注。系统性红斑狼疮 ( systemic lupus erythematosus,SLE）是一种是受环境、遗传等多种因素共同作用下导致多组织、多器官损害的特异性自身免疫性结缔组织病。 在诸多因素中，维生素D 代谢通路基因对于其发生发展起十分重要的调控作用。遗传多态性是个体差异和疾病发生发展的遗 传基础, 单核苷酸多态性是最常见的遗传变异。多篇文献表明，维生素 D 代谢通路基因单核苷酸多态性与 SLE 有相关联性。 于是论文通过查阅相关文献, 从基因层面出发, 对维生素代谢通路基因单核苷酸多态性与 SLE 发生发展相关的研究进展进行 归纳总结，为未来治疗提供新思路。

\section{关键词}

维生素 D 代谢通路; 单核苷酸多态性; 系统性红斑狼疮

\section{【基金项目】大学生创新创业国家级训练项目（项目编号: 202011810019)。 \\ 【第一作者】李赛嘉, 男, 本科学历, 通讯邮箱: 1418951238 @ad.com。}

【通讯作者】周代锋, 男, 教授, 从事疾病发生的分子遗传 机制研究。 


\section{1 引言}

近年来有研究显示, SLE 患者血清维生素 D 缺乏 ${ }^{[1]}$ $\mathrm{Gao}$ 等对维生素 D 与 SLE 的关系进行了研究发现，有 $62.81 \%$ 的患者体内维生素 D 不足, 有 $34.71 \%$ 的患者体内维生素 D 严重缺乏, 这说明维生素 D 缺乏会明显增加 SLE 发病率 ${ }^{[2]}$ 。 一些动物实验也证明适量维生素 D 可以起到降低狼疮鼠尿蛋 白含量、关节功能受损及减轻肾功能所受到的损害 ${ }^{[3]}$ 。在遗 传层次研究中, 罗雄燕、刘军麟等人对维生素 $\mathrm{D}$ 代谢通路基 因遗传多态性与 SLE进行了进一步的研究 ${ }^{[4-5]}$, 在国际文献中, Ozaki、Huang 等人也进行了相关性的研究 ${ }^{[6-7]}$ 。论文针对维 生素 D 代谢通路相关基过来因遗传多态性与 SLE 发生发展的 机制相关研究进展作一综述。

\section{2 系统性红斑狼疮 (SLE) 相关研究进展}

\section{1 发病机制}

系统性红斑狼疮 ( systemic lupus erythematosus, SLE) 是 好发于育龄期女性的自身免疫系统疾病 ${ }^{[8]}$, 具有发病率高, 多系统、多器官受累，反复发作等发病特点 ${ }^{[9-10]}$ 。目前，其 发病机制尚不完全清楚, 主要包括免疫复合物形成从而累积 1 造成多器官损伤、自身抗体的产生、 $\mathrm{T}$ 细胞及 B 细胞的过 度活化等免疫系统的调节异常等 ${ }^{[11-13]}$ 。

\section{2 临床表现}

SLE 在临床发病的症状较为复杂, 主要包括呼吸系统受 损、肾损害症状、发热、面部红斑、关节痛等。有些轻症病 例仅有关节痛或面部皮疹, 严重患者早期便出现危及生命的 严重血小板减少、神经精神狼疮、急进性狼疮肾炎和肺泡出 血等症状 ${ }^{[10 、 14] 。 ~}$

\section{3 常用治疗方法}

临床治疗 SLE 的药物主要包括激素、免疫抑制剂等, 被 称为标准治疗 ( stand of care, SoC ), 常以糖皮质激素与泼尼松、 冲击量甲松龙联用，在 SLE 发病的急性期，大量的使用糖皮 质激素可能会将病人从生死线拉回, 但会出现不良反应 ${ }^{[15]}$ 。

\section{3 维生素 D 代谢通路相关研究进展}

VD 在体内存在的形式多样, 包括 25- 羟维生素 D (25$(\mathrm{OH}) \mathrm{D}) 、 1,25-$ 二 羟 维生素 D (1, 25- ( OH ) 2D ) ${ }^{[16]}$, VD 首先在肝脏中经过 CYP2R1 基因编码的 $25-\alpha$ 差化
酶羟化作用下形成 25- ( OH ) D, 25- 羟维生素 D 被认为 是最能代表人体 VD 总体水平的生物标记物 ${ }^{[17]}$; 后在肾脏中 经过 CYP27B1 基因编码的 1- $\alpha$ 羟化酶羟化形成 ${ }^{[18]}, 1,25-$ $(\mathrm{OH}) 2 \mathrm{D}$ 是 $\mathrm{VD}$ 的活性形式, 1,25- $(\mathrm{OH}) 2 \mathrm{D}$ 在 CYP24A1 基因编码的 24 羟化酶作用下形成 1,24,25-( OH ) 3,24 羟 化酶也将 $25(\mathrm{OH}) \mathrm{D}$ 和 1,25- ( OH ) 2D 进行降解, 起负 反馈调节作用 ${ }^{[19]}$ 。维生素 D 结合蛋白 (VitaminD Binding protein,VDBP ) 由 VDBP 基因编辑, 与维生素 D 结合, 促 进维生素 $\mathrm{D}$ 在肝脏和肾脏转运。1,25- $(\mathrm{OH}) 2 \mathrm{D}$ 与维生素 $\mathrm{D}$ 受体 (VitaminD receptor,VDR) 结合后发挥生物效应, 维 生素 $\mathrm{D}$ 受体由 VDR 基因编码。维生素 $\mathrm{D}$ 代谢通路基因是指 $\mathrm{VD}$ 转化成 1,25-( $\mathrm{OH}) 2 \mathrm{D}$ 过程中起调控作用的基因, 主要 包括 CYP2R1、CYP27B1、CYP24A1、VDBP、VDR五个基因， 维生素 $\mathrm{D}$ 代谢通路基因的异常表达可能影响维生素 $\mathrm{D}$ 的水平, 从而影响生物效能的发挥 ${ }^{[20]}$ 。

\section{4 维生素 D 代谢通路基因多态性与 SLE}

\subsection{CYP2R1 基因多态性与 SLE}

CYP2R1 基因位置处于人体 11 号染色体 $\mathrm{p} 15.2$, 跨度 达 $15.5 \mathrm{~kb}$, 有 5 个外显子 ${ }^{[21]}$ 。CYP2R1 基因编码维生素 D 的 25-羟化酶（CYP2R1），存在在肝脏中的微粒体细胞色素 $\mathrm{P} 450$, 是 P450 家族的 2 亚家族的成员，由 501 个氨基酸构 成 ${ }^{[22]}$ 。CYP2R1 的主要作用是在肝脏进行维生素 D 的 25 位 羟化, 生成 $25-$ 羟维生素 D $(25-(0 \mathrm{H}) \mathrm{D})$, 为维生素 D 代谢循环中的主要产物。根据全基因组关联研究 (GenomeWide Association Studies, GWAS) 确定 CYP2R1 的单核苷 酸多态性与维生素 D 水平相关, 之前的一些研究也显示了 CYP2R1 变异体与 25- $(0 \mathrm{H}) \mathrm{D}$ 水平之间的显著相关性 ${ }^{[23-24]}$ 。 从 Wang 等研究结论中也证实了 CYP2R1 和 CYP27A1 均对 维生素 D 的 25- 差基化起作用 ${ }^{[23]}$,但 CYP2R 基因起主导作用, CYP27A1 是合成 $25-(0 H) D$ 的次要因素。

大量研究表明, CYP2R1 的单核苷酸多态性与许多疾病显 著相关。非典型的维生素 $\mathrm{D}$ 缺乏佝偻病的发生与 CYP2R1 基 因突变有关, Xu 等研究发现 CYP2R1 的位点 rs10766197 与维 吾尔族人群血清维生素 D 含量低有明显的相关性 ${ }^{[25]}$ 。Wang 等 通过对比研究证实 CYP2R1 的位点 rs10766197 与 II 型糖尿病 患者血清 25- $(0 \mathrm{H}) \mathrm{D}$ 水平显著相关 ${ }^{[26]}$, 同时 CYP2R1 的基 因多态性发现与 I 型糖尿病的易感性有显著关联, CYP2R1 的 
低表达可能与 I 型糖尿病患者血液中的 25- ( OH ) D 浓度降低 有关。CYP2R1 所诱导产生的产物 25- $(0 \mathrm{H}) \mathrm{D}$ 与多种癌症的 易感性相关, 包括胰腺癌、乳腺癌等。Sheng 等通过大量研究 分析发现高表达的 CYP2R1 基因与乳腺癌无复发性生存率显 著相关 ${ }^{[27]}$ 。CYP2R1 基因中的位点 rs10741657、rs2060793、 rs12794714 与胰腺癌易感性相关达 20\% 30\%。在 GWAS 中, CYP2R1 的这些 SNP 位点与 25- $(0 \mathrm{H}) \mathrm{D}$ 水平显著相关。

大量 CYP2R1 基因的位点除了证实与这些疾病有关, 同 样说明了与 25- $(0 \mathrm{H})$ D 水平的关联性。25- $(0 \mathrm{H}) \mathrm{D}$ 是维 生素 D 在血液中存在的主要形式, 目前临床上测定机体维生 素 D 的水平多通过检测血清 225- ( $0 \mathrm{H}) \mathrm{D}$ 来反映 ${ }^{[28]}$ 。有研 究表明 SLE 患者广泛存在血清 25- $(0 \mathrm{H})$ D 水平不足或缺乏 的问题 ${ }^{[29]}$, Squance 等的研究发现, 将 80 例 SLE 患者和 41 名健康正常体检者的血清进行对比, SLE 患者血清中的 25$(0 \mathrm{H}) \mathrm{D}$ 的水平明显低于正常健康人群 ${ }^{[30]}$ 。血清 $25-(0 \mathrm{H})$ D 水平在 SLE 发病中起着重要作用, 与 SLE 发病状态相关, 可作为临床指标判断 SLE 疾病的严重程度 ${ }^{[35]}$ 。有研究发现 SLE 患者 25- $(0 \mathrm{H})$ D 水平低于 B 细胞的高度活化和 IFN $-\alpha$ 信号高表达有关, 同时也与高抗 dsDNA 和免疫球蛋白有关 ${ }^{[31]}$ 。 这一发现证明了 25- $(0 \mathrm{H})$ D 水平低可能触发自身抗体产生, 从而增加了个体自身免疫疾病发展的风险。在此基础上, 可 以得出, CYP2R1 基因通过影响维生素 D 代谢通路中的维生 素 D 肝脏 25 位羟化, 从而影响血液中 25- $(0 \mathrm{H}) \mathrm{D}$ 水平, 进一步影响 SLE。

\subsection{VDBP 基因多态性与 SLE}

维生素 D 结合蛋白 ( Vitamin D Binding Protein,VDBP ) 由 VDBP 基因编码, VDBP 是一种能发挥多种作用的血浆 蛋白, 在体内多种组织内合成, 但合成场所主要在肝脏中。 1959 年被成功分离出, 最初因其免疫学特征被称为族特异性 成分 (group-specific conponent) - GC 球蛋白 ${ }^{[32]}$ 。后来经多 次研究验证发现 GC 蛋白与 VDBP 为同一种蛋白 ${ }^{[33]}$ 。VDBP 的编码基因 $\mathrm{GC}$ 定位于 4 号染色体的长臂 q11-q12, 该基因 目前发现有 2000 多个 SNP 是, VDBP 与维生素 D 之间有着 密切的联系, VDBP 在维持血清维生素 D 的含量、调整维生 素 D 生物利用度、维生素 D 活性、末端组织对维生素 D 的 反应中发挥极其重要的作用 ${ }^{[34]}$ 。其中, VDBP 对维生素 D 胞 饮起着非常重要作用, 且它的基因多态性会影响维生素 D 水
平及其活性 ${ }^{[35]}$ 。此外有研究表明, VDBP 在维生素 D 代谢通 路中也发挥着非常重要的作用, 它的多态性与免疫应答、结 合维生素 $\mathrm{D}$ 和衍生物的能力有关。

其中中国和国际上对 VDBP 基因单核苷酸多态性位点与 疾病的关联性研究较多 ${ }^{[36]}$ 。VDBP 与肺病、肝病、肥胖、骨 组织疾病、糖尿病等多种疾病之间存在关联性 ${ }^{[37-39]}$ 。目前中 国和国际上对于 VDBP 编码基因的研究大多集中在 rs2282679 $(\mathrm{A} / \mathrm{C}) 、 \operatorname{rs} 45889(\mathrm{C} / \mathrm{A}) 、 \operatorname{rs} 7041(\mathrm{~T} / \mathrm{G})$ 这三个位点, 研究发现 rs2282679 ( A/C) 多态性与维生素 D 水平有关联 性 ${ }^{[40-42]}$, 且 SNP 位点 rs2282679 与骨代谢疾病、肥胖、心肺 等疾病有相关联性 ${ }^{[37]}$ 。Regulla 等研究发现 VDBP 基因多态 性与 Graves 的疾病有关联性 ${ }^{[43]}$ 。王高帅等人研究发现 VDBP 的 SNP 位点 rs7041 与肥胖有密切关系 ${ }^{[44]}$ 。但目前, VDBP 基 因多态性在 SLE 发病机制中的作用尚不清楚, 且尚无大样本 关于 SLE 患者与 VDBP 基因多态性相关联的临床研究及报道, 所以仍有待后续实验验证。

\subsection{CYP27B1 基因多态性与 SLE}

CYP27B1 基因是 $1-\alpha$ 羟化酶的编码基因, 存在于人类 12 号染色体长臂 (12q13.1-q13.3)，由 9 个外显子和 8 个内 含子组成, 为单拷贝基因。其 $\mathrm{cDNA}$ 全长为 $4.8 \mathrm{~kb}$, 编码了含 508 个氨基酸的多肽 ${ }^{[45-46]}$, 即 $\mathrm{P} 450$ 酶家族的成员, 编码 $1-\alpha$ 着化酶。

$1-\alpha$ 着化酶是催化 $25-(0 \mathrm{H}) \mathrm{D}$ 形成 $1,25-(0 \mathrm{H}) 2 \mathrm{D}$ 的限速酶, 它的主要功能是在肾脏近曲小管和直精细管催化 25- ( $0 \mathrm{H}) \mathrm{D}$ 的羟化及活化作用, 将其转化为其活性形式 1,25$(0 \mathrm{H}) 2 \mathrm{D}^{[47-48]}$ 。Panda、张增利等人均发现 $1-\alpha$ 羟化酶活性 的变化与血浆和局部的 $1,25-(0 \mathrm{H}) 2 \mathrm{D}$ 水平和免疫系统功能 紊乱有关联性, 在 1- $\alpha$ 着弪化酶基因（CYP27B1）靶向切除的 动物体内不存在活性维生素 $\mathrm{D}^{[49-50]}$ 。多篇文献报道, 1,25- $(0 \mathrm{H})$ $2 \mathrm{D}$ 对 $\mathrm{T}$ 细胞和 $\mathrm{B}$ 细胞均有直接影响, 不仅促进各种炎症的 细胞因子产生，还诱导调节性 $\mathrm{T}$ 细胞参与一种 “关闭” 的炎 症反应。在此基础上, 有研究表明 ${ }^{[51]}$, 维生素 $\mathrm{D}$ 主要是通过 增加调节性 $\mathrm{T}$ 细胞数量和产生抗增殖作用在系统性红斑狼疮 中, 因此从此可得出 CYP27B1 单核苷酸多态性与系统性红斑 狼疮的发生发展有着密切的联系。

\subsection{VDR 基因多态性与 SLE}

$\mathrm{VDR}$ 是一种 $50 \mathrm{kDa}$ 的核受体，属于类固醇受体家族的 
第 2 类, 与视黄酸受体和促甲状腺激素类似 ${ }^{[52]}$ 。1,25- $(0 \mathrm{H})$ $2 \mathrm{D}$ 与核 VDR 基因组结合, 并通过调节某些基因的转录来决 定基因组反应 ${ }^{[53]}$ 。VDR 由位于 12 号染色体 $12 q 13.1$ 位置的基 因合成, 称为 VDR 基因 ${ }^{[54]}$ 。该基因基本上由 9 个外显子组成, 它们分布在 5, 启动子和 3 , 调控区。在后一个区域, 有一个 长长的 3 ' 未翻译区域, 称为 3 ' 未翻译区域, 参与基因表达 的调节, 特别是通过调节信使 RNA 的稳定性。VDR 基因在 3' 翻译区外显子 2 和 9 之间的启动子区, 尤其是外显子 $1 \mathrm{f}$ 和 $\mathrm{c}$ 附近的启动子区出现了一些多态性 ${ }^{[55]}$ 。多态性 BsmI 位于内含 子 8 和腺嘌呤 - 鸟嘌呤取代结果 $(\mathrm{A}-\mathrm{G})^{[56]}$ 。ApaI 和 TaqI 多 态性分布在该区域 $3^{\prime}$ 的基因。多态性 ApaI 定义为内含子 8 中 的胸腺嘧啶取代 ( T-G), 而多态性 TaqI 定义为胞嘧啶 - 胸 腺嘧啶取代 $(\mathrm{C}-\mathrm{T})$, 导致密码子交换 $(\mathrm{ATC} \rightarrow \mathrm{ATT})$, 但 维持相同的异亮氨酸氨基酸 ${ }^{[57]}$ 。这些多态性的功能相关性与 信使 RNA 的稳定性增强有关 ${ }^{[58]}$ 。FokI 多态性是由内含子 1 与 外显子 2 交界处的胞嘧啶 - 胸腺嘧啶 $(\mathrm{C}-\mathrm{T}$ ) 的取代引起的, 产生了一个额外的起始密码子 $(\mathrm{ACG} \rightarrow \mathrm{ATG})$, 三个靠近转 录起始位点的密码子。这种多态性可以被认为是一个独立的 遗传标记, 因为它似乎并没有与其他 VDR 基因多态性在连锁 不平衡。FokI 的出现变体, 定义为 $\mathrm{f}$ ( ATG 密码子) 的变异 使 VDR 蛋白完全生成 ( 427 个氨基酸) ${ }^{[59]}$, 而定义为 f ( 密码 子 GCA ) 的变异 FokI 从另一个位点开始翻译, 合成一个含有 少 3 个氨基酸 ( 424 个氨基酸) 的稍短的 VDR 蛋白。体外研 究表明, 短蛋白似乎比长蛋白具有更高的转录活性 ${ }^{[60]}$ 。这可 能会增加 VDR 的功能, 从而改变维生素 D 在不同细胞和组织 中的作用。FokI 多态性对免疫特异性转录因子在淋巴细胞增 殖和免疫细胞蛋白合成中的转录活性的影响表明该多态性参 与了免疫调节与免疫调节的多态性 ${ }^{[10]}$ 。

VDR 基因 SNP 在维生素 D 转运和代谢途径中与支气管 哮喘、肺结核、变应性鼻炎等呼吸系统疾病及系统性红斑狼疮、 结节性硬化、痛风、帕金森病等疾病易感性有关 ${ }^{[61-63]}$ 。

有大量学者进行了有关 VDR 基因 BsmI 多态性与 SLE 的关系的实验。 2000 年, 一项对 58 名日本 SLE 患者的研究 证明, 与健康对照相比, B/B 基因型出现的频率更高 (15.5 比 $5.7 \%, \mathrm{p}<0.0001$ ）。此外, 更高频率的基因型 $\mathrm{b} / \mathrm{b}$ 在肾 病患者肾病综合症 $(61.5 \%$ 和 $35.7 \%, \mathrm{p}<0.0034)$ 被发现 ${ }^{[64]}$ 。 在 2002 年, 中国作者研究了 47 个系统性红斑狼疮患者和 90 名健康对照, 发现更高的 $\mathrm{b}$ 等位基因频率在例( 39.4\% 和 8.3\%,
$\mathrm{OR}=0.74, \mathrm{p}<0.0001)^{[65]}$ 。2006 年和 2010 年, 分别对 101 名泰国患者和 60 名伊朗患者的研究发现, BsmI 多态性与 SLE 或该疾病的临床和实验室表现没有关联。2002 年, 一项 研究评估了 52 例 SLE 患者和 90 例健康对照的 FokI 多态性, 发现等位基因和基因型频率没有显著差异 ${ }^{[66]}$ 。2010 年, 一项 荟萃分析发表, 结果 FokI 多态性不显著。不过, 由于纳入的 研究数量较少, 这些结果应当谨慎解释, 而且这些结果仅适 用于欧洲和亚洲族裔 ${ }^{[67]}$ 。在巴西进行的一项病例对照研究也 调查了 195 名欧洲或非洲源性患者和 201 名对照患者的 BsmI 和 FokI VDR 基因多形态与 SLE 易感性之间的关系。结果显 示, BsmI 和 FokI VDR 基因多态性与 SLE 易感性之间没有关 联。在该实验的研究中发现, SLE 患者血清 25- ( OH ) D 的 平均水平为 $25.51 \pm 11.43 \mathrm{ng} / \mathrm{ml}$ 。 $\mathrm{f} / \mathrm{f}$ 基因型患者 $25-(0 \mathrm{H})$ $\mathrm{D}$ 水平显著高于 f/f 基因型患者（31.6 $14.1 \mathrm{vs} 23.0 \pm 9.2 \mathrm{ng}$ / $\mathrm{ml}, \mathrm{p}<0.004$ ）。虽然 FokI 多态性与 SLE 无显著相关性, 但作者认为该多态性对 SLE 患者的维生素 D 代谢有重要影响 ${ }^{[68]}$, 最近在中国患者中又发现了 Bsmi 和 FokI VDR 多态性。 SLE 患者中纯合子 F/F 的频率高于对照组（42.8 对 25.4\%, $p=0.001$ )。纯合子 $F / F$ 和杂合子 $F / F$ 的 SLE 患者血清炎、 抗 dsdna 抗体、抗 $s m$ 抗体和抗组蛋白抗体均高于纯合子 $F / F$ 的 SLE 患者。SLE 患者 B 等位基因的频率明显增加，B 等位 基因与狼疮肾炎有关，也与抗核小体抗体的产生有关 ${ }^{[69]}$ 。

\section{5 结论}

目前, 已有大量研究探索了维生素 D 缺乏与 SLE 疾病 发生、发展等的联系, 但对其机制研究大多停留在细胞层次, 少数涉及遗传层次。但随着对 SLE研究的深入, 我们不难发现, 基因的遗传多态性对 SLE 的发生发展起着不可忽视的作用。 而维生素 D 代谢通路作为维生素 D 重要的生成途径, 其基因 的缺失与否也直接影响体内维生素 $\mathrm{D}$ 的水平, 从而进一步影 响某些疾病, 特别是自身免疫性疾病 (例如 SLE) 的发生发展。 由此, 研究 SLE 基因遗传多态性成为新的热门并且为其前期 预防与后续相关治疗提供一定的帮助, 有望成为 SLE 研究治 疗的新方向。

\section{参考文献}

[1] 黄雨茜, 张浩, 张双, 等. 维生素 $\mathrm{D}$ 受体与 $\mathrm{MCP}-1$ 在系统性红斑 狼疮患者中的表达及意义 [J]. 南方医科大学学报, 2020(01):99-103.

[2] GAO CC,LIU SY,WU ZZ,et al.Severe vitamin D deficiency increases 
the risk for moderate to severe disease activity in Chinese patients with SLE [J].Lupus,2016(11):1224-1229.

[3] MARINHOA,TAVEIRAM,VASCONCELOSC.Topics on vitamin D in systemic lupus erythematosus: analysis of evidence and critical literature ZZreview[J].Immunologic Research,2017(02):495-511.

[4] 罗雄燕, 陈龙, 杨明辉, 等. 维生素 D 受体各位点基因多态性与系 统性红斑狼疮的相关性研究 [A]. 第 17 次全国风湿病学学术会议 论文集 [C]. 中华医学会, 中华医学会风湿病学分会, 2012 .

[5] 刘军麟.PDCD1 基因与系统性红斑狼疮以及维生素 D 受体基因与 银屑病关联研究的 Meta- 分析 [D]. 合肥 : 安徽医科大学, 2008.

[6] Ozaki Y,Nomura S,Nagahama M,et al.Viamin-D receptor genotype and renal disorder in Japanese patients with systemic lupus erytheMatosu [J].Nephron.2000(01):86-91.

[7] Huang CM,Wu MC,Wu JY, et al.No association of vitamin D receptor gene start codon fokl polymorphisms in Chinese patients with systemic lupus erythematosus [J].Rheumatol.2002(06):1211.

[8] Mohan C,Putterman C.Genetics and pathogenesis of systemic lupus erythematosus and lupus nephritis[J].Nat Rev Nephrol,2015(06):329-341.

[9] Ahmadpoor P,Dalili N,Rostami M.An update on pathogenesis of systemic lupus erythematosus[J].Iran J Kidney Dis, 2014(03):171184.

[10] 谢长好, 李志军. 系统性红斑狼疮的诊断与治疗 [J]. 中华全科医 学, 2020(04):527-528.

[11] Sanz I.New Perspectives in Rheumatology:May You Live in Interesting Times:Challenges and Opportunities in Lupus Research[J]. Arthritis Rheumatol,2017(08):1552-1559.

[12] Dorner T,Lipsky PE.Beyond pan-B-cell-directed therapy-new avenues and insights into the pathogenesis of SLE[J].Nat Rev Rheumatol,2016(11):645-657.

[13] 罗春华, 李鹏, 李乾元, 等. 系统性红斑狼疮患者血清细胞因子水 平的变化及临床意义 [J]. 医学综述, 202(08):1641-1644+1649.

[14] Ospina FE,Echeverri A,Zambrano D,et al.Distinguishing infections vs flares in patients with systemic lupus erythematosus[J].Rheumatology( Oxford ),2017(01):46-54.

[15] Kamal A,Khamashta M.The efficacy of novel B cell biologics as the future of SLE treatment: a review[J].Autoimmun Rev,2014(13):10941101.
[16] 曾琴, 袁晶晶, 谢忠建. 维生素 D 与甲状腺癌的研究现状 [J]. 中华 内分必代谢杂志 ,2017(06):525-528.

[17] 武莉莉, 刘春丽. 维生素 $\mathrm{D}$ 与呼吸系统感染性疾病 $[\mathrm{J}]$. 华夏医 学, 2018(02):159-163.

[18] ChenSai-Ming;ZhouXiao-Liu;LIZhi-Lu,et al.Association between the genetic variation of vitamin D binding protein gene and chronic sinusitis of elder in Hainan province[J].Chinese Journal of Gerontology,2019(11):2694-2697.

[19] 王崧成. 维生素 D 代谢通路基因多态性、拷贝数及甲基化变异与 2 型糖尿病的关系 [D]. 郑州: 郑州大学, 2018 .

[20] 李嘉恒. 维生素 D 水平与其代谢通路相关基因表达及胚胎质量关 系的研究 [D]. 郑州大学, 2019 夏医学, 2018(02):159-163.

[21] Cheng JB,Motola DL,Mangelsdorf DJ,et al.De-orphanization of cytochrome P450 2R1: a microsomal vitamin D 25-hydroxilase[J].J Biol Chem,2003(39):38084-38093.

[22] 李玲, 赵慧佳, 陈涁尧, 等.CYP2R1 的遗传多态性及其个体化治 疗应用 [J]. 中国临床药理学与治疗学, 2019(09):1053-1059.

[23] Wang TJ,Zhang F,Richards JB, et al.Common genetic determinants of vitamin D insufficiency: a genome-wide association study[J]. Lancet,2010(9736):180-188.

[24] Cheng JB,Motola DL,Mangelsdorf DJ,et al.De-orphanization of cytochrome P450 2R1: a microsomal vitamin D 25-hydroxilase[J].J Biol Chem,2003(39):38084-38093.

[25] Xu X,Mao J,Zhang M,et al.Vitamin D deficiency in Uygurs and Kazaks is associated with polymorphisms in CYP2R1 and DHCR7/ NADSYN1 genes[J].Med Sci Monit,2015(21):1960-1968.

[26] Wang Y,Yu F,Yu S,et al.Triangular relationship between CYP2R1 gene polymorphism,serum 25(OH)D3 levels and T2DM in a Chinese rural population[J].Gene,2018(18):30863-30871.

[27]Sheng L,Callen DF, Turner AG.Vitamin D3 signaling and breast cancer:Insights from transgenic mouse models[J].J Steroid Biochem Mol Biol,2018(178):348-353.

[28] 张永锋, 郑毅. 初发系统性红斑狼疮患者血清 25-着弪-维生 素 $\mathrm{D}$ 和维生素 $\mathrm{D}$ 抗体水平变化及意义 $[\mathrm{J}]$. 中华风湿病学杂 志 ,2012(10):661-664

[29] Schoindre Y,Jallouli M,Tanguy ML, et al.Lower vitamin D levels are associated with higher systemic lupus erythematosus activity, but not predictive of disease flare-up.[J].Lupus science \& 
medicine, 2014(01):27.

[30] 陈斯亮, 符青松, 罗莞超, 等. 25- 羟维生素 D3 与 IL-10 在 系统性红斑狼疮中的临床应用研究 [J]. 分子诊断与治疗杂 志 ,2018(03):180-183+195.

[31] Szodoray P,Tarr T,Bazso A,et al.The immunopathological role of vitamin D in patients with SLE: data from a single centre registry in Hungary[J].Scandinavian Journal of Rheumatology,2011(02): 122.

[32] HIRSCHFELD J,BECKMAN L.A new group-specific serum system (Gc-groups) in relation to blood and serum groups.[J].Acta genetica et statistica medica, 1960(10):48-53.

[33] Daiger S P,Schanfield M S,Cavalli-Sforza L L.Group-specific component $(\mathrm{Gc})$ proteins bind vitamin D and 25-hydroxyvitamin $\mathrm{D}[\mathrm{J}]$.Proceedings of the National Academy of Sciences of the United States of America .1975(06):2076-2080

[34] 刘凤英, 任伟, 张素华. 维生素 D 结合蛋白基因多态性与 2 型糖 尿病 [J]. 重庆医学, 2004(06):923-925.

[35] Wilson PW ,Cupples LA,Meigs JB.et al.Genome scan for impaired glycemic status:results from the Framingham Heart Study[ J] .Diabetes, 1997(01):76A.

[36] 陈赛明, 黄静, 周小柳, 等. 维生素 D 结合蛋白基因多态性与海南 慢性变应性鼻炎的关联性研究 [J]. 海南医学院学报,2018(22):20192022.

[37] 杨骏杰, 张岩, 李晨光, 等. 维生素 D 结合蛋白及其基因多态性的 研究进展 [J]. 上海医学, 2019(05):308-313.

[38] Kitanaka S,Isojima T,Takaki M,et al.Association of vitamin D -related genepoly morphisms with manifestation of vitamin D deficiency in children [J].Endocr J,2012(11):1007-1014.

[39] Lester E,Skinner R K,Wills M R. Seasonal variation in serum 25-hydroxyvitamin D in the elderly in Britain [J]. Laneet,1977(8019):979-980.

[40] Cleve H,Constans J.The mutants of the vitamin-D-binding protein: more than 120 variants of the GC/DBP system.[J].Vox Sanguinis, 1988(04):215-25.

[41] Viau,Constans, Debray,et al.Isolation and characterization of the $\mathrm{O}-$ glycan chain of the human vitamin-D binding protein $[\mathrm{J}]$. Biochemical and Biophysical Research Communicatio ns, 1983(01):324-331.

[42] Lisa B.Signorello,Jiajun Shi, Qiuyin Cai,et al.Common Variation in
Vitamin D Pathway Genes Predicts Circulating 25-Hydroxyvitamin D Levels among African Americans[J].PLOS ONE,2011(12):13-14.

[43] Pani Michael A,Regulla Karoline,Segni Maria,et al.A polymorphism within the vitamin D-binding protein gene is associated with Graves' disease but not with Hashimoto's thyroiditis. [J].The Journal of Clinical Endocrinology \& Metabolism,2002(06):2564.

[44] 王重建, 王高帅, 李玉倩, 等.DBP 基因 rs7041 位点多态性与汉族 人群肥胖易感性的关系 [J]. 郑州大学学报 (医学版),2015(03):331334.

[45] Portale AA, Miller WL.Human 25-hydroxyvitamin-Dlalphahydroxylase: cloning,mutations, and gene expression [J].Pediatr Nephrol,2000(07):620-625.

[46] 杨静.CYP27B1 基因多态性与自身免疫性甲状腺疾病易感性的关 联研究 [D]. 重庆: 重庆医科大学, 2008 .

[47] Hewison M,Zehnder D,Bland R, et al.1-Hydroxylase and the action of vitamin D[J].Journal of Molecular Endocrinology.2000(25):141-148.

[48] Miller WL,Portale AA.Vitamin D 1-alpha-hydroxylase[J].Trends in Endocrinology and Metabolism.2000(11):315-319.

[49] Panda DK, Miao D,Tremblay ML,et al.Targeted ablation of the 25-hydroxy vitamin D 1alpha-hydroxylase enzyme:evidence for skeletal,reproductive, and immune dysfunction [J].Proc Natl Acad Sci MSA.2001(98): 7498-7503.

[50] 张增利, 李冰燕, 童建. 利用基因敲除小鼠研究维生素 $\mathrm{D}$ 在免疫 功能发育中的作用 [J]. 中华微生物学和免疫学杂志, 2007(03):260263.

[51] 王瑞, 王丹, 艾娇, 等. 维生素 D 对系统性红斑狼疮的影响 [J]. 医 学综述, 2019(21):4251-4256.

[52] DeLuca Hector F.Overview of general physiologic features and functions of vitamin D.[J].The American journal of clinical nutrition, 2004(06):48.

[53] Yamada Sachiko,Makishima Makoto.Structure-activity relationship of nonsecosteroidal vitamin D receptor modulators. [J].Trends in pharmacological sciences, 2014(07):35-37.

[54] Miyamoto K,Kesterson R A,Yamamoto H,et al.Structural organization of the human vitamin $\mathrm{D}$ receptor chromosomal gene and its promoter. [J].Molecular endocrinology (Baltimore,Md.),1997(08):132-135.

[55] André G.Uitterlinden, Yue Fang,Joyce B.J.van Meurs, et 
al.Genetics and biology of vitamin D receptor polymorphisms[J]. Gene,2004(02):721.

[56] Morrison N A,Yeoman R,Kelly P J,et al.Contribution of trans-acting factor alleles to normal physiological variability: vitamin D receptor gene polymorphism and circulating osteocalcin.[J].Proceedings of the National Academy of Sciences of the United States of America, 1992(15):87

[57] Faraco J H,Morrison N A,Baker A, et al.ApaI dimorphism at the human vitamin D receptor gene locus. [J].Nucleic acids research, 1989(05):312-314.

[58] Morrison N A,Qi J C,Tokita A,et al.Prediction of bone density from vitamin D receptor alleles.[J].Nature,1994(6460):367.

[59] Coleman Gross,T.Ross Eccleshall,Peter J.Malloy,et al.The presence of a polymorphism at the translation initiation site of the vitamin $\mathrm{D}$ receptor gene is associated with low bone mineral density in postmenopausal mexican-American women[J].Journal of Bone and Mineral Research,1996(12): 521-523.

[60] Arai H,Miyamoto K, Taketani Y,et al.A vitamin D receptor gene polymorphism in the translation initiation codon: effect on protein activity and relation to bone mineral density in Japanese women. $[\mathrm{J}]$. Journal of bone and mineral research : the official journal of the American Society for Bone and Mineral Research,1997(06):37.

[61] Evelyne van Etten,Lieve Verlinden, Annapaula Giulietti,et al.The vitamin D receptor gene Fok I polymorphism: Functional impact on the immune system[J].European Journal of Immunology, 2007(02):78-79.

[62] Milena Despotovic, Tatjana Jevtovic Stoimenov,Ivana Stankovic,et al.Vitamin D Receptor Gene Polymorphisms in Serbian Patients
With Bronchial Asthma: A Case-Control Study[J].Journal of Cellular Biochemistry,2017(11):118.

[63] Harishankar Mahto, Rina Tripathy,et al.Association between vitamin D receptor polymorphisms and systemic lupus erythematosus in an Indian cohort[J].International Journal of Rheumatic Diseases, 2018(02):113-114.

[64] Pan Zhipeng,Chen Mengya,Hu Xingxing,et al.Associations between VDR gene polymorphisms and colorectal cancer susceptibility: an updated meta-analysis based on 39 case-control studies. [J]. Oncotarget,2018(16):46.

[65] Ozaki Y,Nomura S,Nagahama M,et al.Vitamin-D receptor genotype and renal disorder in Japanese patients with systemic lupus erythematosus[J].Nephron,85(01):86-91

[66] C-M Huang.Association of vitamin D receptor gene BsmI polymorphisms in Chinese patients with systemic lupus erythematosus, 2002(01):31-34.

[67] Huang CM,Wu MC,Wu JY, et al .No association of vitamin D receptor gene start codon fok 1 polymorphisms in Chinese patients with systemic lupus erythematosus, 2002(06):1211-1213.

[68] Lee YH,Bae SC,Choi SJ,et al.Associations between vitamin D receptor polymorphisms and susceptibility to rheumatoid arthritis and systemic lupus erythematosus: a metaanalysis [J].2011(38):36433651.

[69] Monticielo OA,Brenol JC,Chies JA, et al.The role of BsmI and FokI vitamin D receptor gene polymorphisms and serum 25-hydroxyvitamin D in Brazilian patients with systemic lupus erythematosus[J]. 2012(01):43-52. 